\title{
Optical observations geomagnetically conjugate to sprite-producing lightning discharges
}

\author{
R. A. Marshall ${ }^{1}$, U. S. Inan ${ }^{1}$, T. Neubert ${ }^{2}$, A. Hughes ${ }^{3}$, G. Sátori ${ }^{4}$, J. Bór ${ }^{4}$, A. Collier ${ }^{3}$, and T. H. Allin ${ }^{5}$ \\ ${ }^{1}$ STARLab, Stanford University, Stanford, CA, USA \\ ${ }^{2}$ Danish Space Research Institute, Copenhagen, Denmark \\ ${ }^{3}$ University of KwaZulu-Natal, Durban, South Africa \\ ${ }^{4}$ Geodetic and Geophysical Research Institute, Sopron, Hungary \\ ${ }^{5}$ Danish Technical University, Lyngby, Denmark
}

Received: 5 July 2004 - Revised: 12 March 2005 - Accepted: 25 March 2005 - Published: 15 September 2005

\begin{abstract}
Theoretical studies have predicted that large positive cloud-to-ground discharges can trigger a runaway avalanche process of relativistic electrons, forming a geomagnetically trapped electron beam. The beam may undergo pitch angle and energy scattering during its traverse of the Earth's magnetosphere, with a small percentage of electrons remaining in the loss cone and precipitating in the magnetically conjugate atmosphere. In particular, $N_{2} 1 P$ and $N_{2}^{+} 1 N$ optical emissions are expected to be observable. In July and August 2003, an attempt was made to detect these optical emissions, called "conjugate sprites", in correlation with sprite observations in Europe near $L \simeq 1.78$. Sprite observations were made from the Observatoire du Pic du Midi (OMP) in the French Pyrenées, and VLF receivers were installed in Europe to detect causative sferics and ionospheric disturbances associated with sprites. In the Southern Hemisphere conjugate region, the Wide-angle Array for Sprite Photometry (WASP) was deployed at the South African Astronomical Observatory (SAAO), near Sutherland, South Africa, to observe optical emissions with a field-of-view magnetically conjugate to the Northern Hemisphere observing region. Observations at OMP revealed over 130 documented sprites, with WASP observations covering the conjugate region successfully for 30 of these events. However, no incidences of optical emissions in the conjugate hemisphere were found. Analysis of the conjugate optical data from SAAO, along with ELF energy measurements from Palmer Station, Antarctica, and charge-moment analysis, show that the lightning events during the course of this experiment likely had insufficient intensity to create a relativistic beam.
\end{abstract}

Keywords. Ionosphere (Ionsophere-magnetosphere interactions; Ionospheric disturbances; Instruments and techniques)

Correspondence to: R. A. Marshall

(ram80@stanford.edu)

\section{Introduction}

Detection of terrestrial gamma-rays above active thunderstorms on the Compton Gamma-Ray Observatory (CGRO) has provided evidence of relativistic electrons in the upper atmosphere (Fishman et al., 1994). These gamma-ray flashes result from brehmsstrahlung radiation from relativistic electrons. Several of these gamma-ray flashes were found to be coincident with large positive cloud-to-ground $(+\mathrm{CG})$ lightning flashes below the CGRO pass (Inan et al., 1996), providing evidence that a lightning flash is causatively associated with these relativistic electrons. Further evidence is provided by Dwyer et al. (2004), who observe gamma-rays originating from $6-8 \mathrm{~km}$ altitude associated with rocket-triggered lightning.

A 1-D fully electrodynamic model developed by Lehtinen et al. (2001) predicts the formation of a beam of relativistic electrons propagating away from the thundercloud and into the magnetosphere, where the electron trajectories are confined to magnetic field lines. Following the charge removal by the $+\mathrm{CG}$, a quasi-electrostatic field is established between the cloud and the ionosphere above it. This field accelerates seed relativistic electrons (created by a constant flux of cosmic rays from space), initiating an avalanche process in the upper atmosphere. The resulting intense beam of electrons is accelerated upwards, generates gamma-ray emissions at altitudes of $\sim 60$ to $70 \mathrm{~km}$, and travels through the lower ionosphere and into the magnetosphere.

Initially, all electrons in the beam are in the loss cone and would thus precipitate in the conjugate hemisphere if no interaction with the cold magnetospheric plasma were to take place. However, the beam may undergo pitch angle scattering and energy degradation during its traverse, through wave-particle resonance interactions, so that only a fraction of electrons remain in the loss cone. In the worst case, when pitch angles are scattered into an isotropic distribution, the number in the loss cone is a function of geomagnetic 

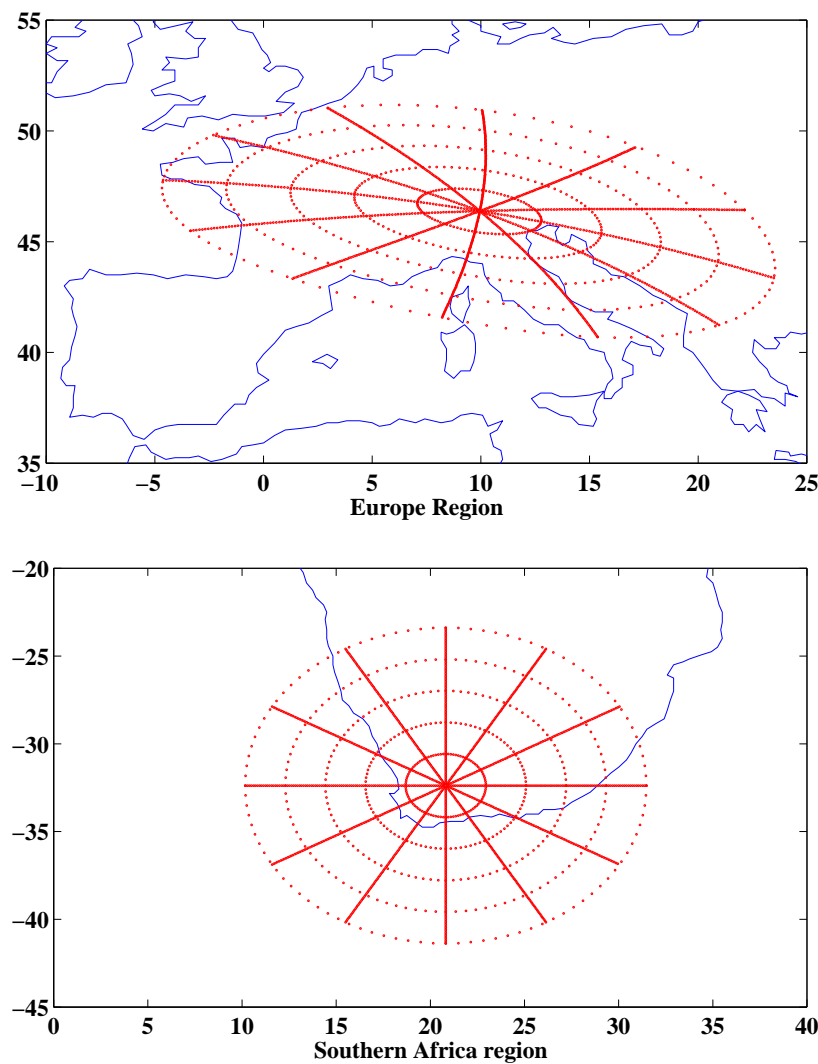

Fig. 1. Regions of interest for conjugate sprite experiment. In Southern Africa region, rings are centered on SAAO and are spaced at $200 \mathrm{~km}$ radius. Each point is magnetically conjugated to the European region.

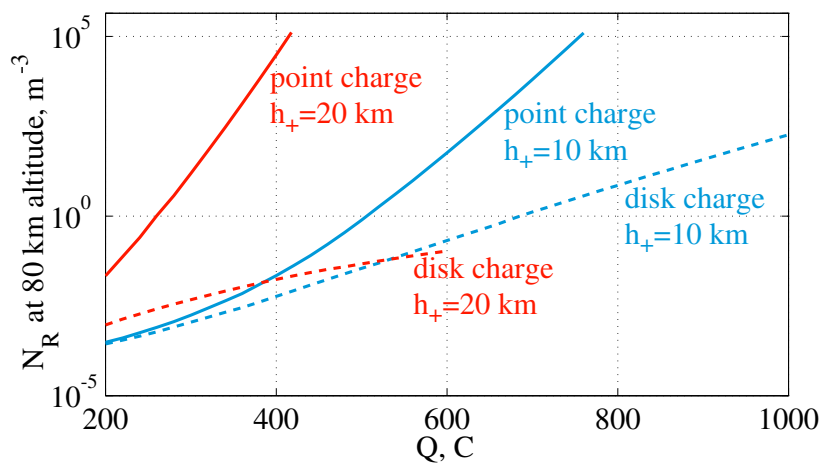

Fig. 2. Charge removed versus injected beam density for point and disc charge configurations, at altitudes of 10 and $20 \mathrm{~km}$. From Lehtinen et al. (2001).

latitude; at latitudes of $30-40 \mathrm{deg}$, this number varies from 10 down to $3 \%$ (Lehtinen et al., 2001). However, even if only $\sim 3$ to $10 \%$ of the electrons precipitate on the conjugate atmosphere, they create detectable emissions via interactions with atmospheric species. For $500 \mathrm{C}$ of charge removed from $15 \mathrm{~km}$ altitude $\left(M_{q}=7500 \mathrm{C}-\mathrm{km}\right)$, consistent with the largest observed gamma-ray fluxes, the injected beam density is $N_{e}=10^{5} \mathrm{~m}^{-3}$, and the predicted emissions should be of a similar magnitude to elves (Lehtinen et al., 2001). While this charge moment is very high compared to observed values (e.g., Cummer and Inan, 2000), it results in a relativistic beam density that is consisted with BATSE gamma-ray flash observations (Lehtinen et al., 2001).

In this paper, we report results of an experimental attempt to detect these optical emissions following large + CG lightning strikes. This experiment is motivated by observations of BATSE terrestrial gamma-ray flashes associated with lightning, which provide real evidence for the existence of relativistic electron beams created by lightning. Sprite observations in the Northern Hemisphere are complemented by photometric measurements taken from South Africa, at the geomagnetically conjugate point to the lightning strikes. Examples of data are presented along with charge moment and ELF energy measurements.

\section{Experimental setup}

The experiment was conducted in July and August 2003 from the South African Astronomical Observatory (SAAO) near Sutherland, South Africa, to observe conjugate signatures of relativistic electron beams. The region of interest of lightning activity lies in the South of France and the Alps, as shown in Fig. 1. These magnetically conjugate regions are ideally suited to the experiment, offering high lightning activity in the local (Europe) region, good observing conditions over land in the conjugate region, mid-magnetic latitudes, and additionally, recent documentation of sprite activity in the local region (Neubert et al., 2001).

Following the model predictions in Lehtinen et al. (2001), we should expect to see emissions of intensity $\sim 1$ MR (one mega-rayleigh; one rayleigh is $10^{6} / 4 \pi$ photons $\mathrm{cm}^{-2} \mathrm{str}^{-1} \mathrm{~nm}^{-1}$ ) due to a point charge removal of $\sim 750 \mathrm{C}$ from $10 \mathrm{~km}$ altitude, lasting about $1 \mathrm{~ms}$. However, as shown in Fig. 2, the relationship between charge removed and beam density injected is highly non-linear. The noise limit of the instrument is about $1 \mathrm{kR}$; this requires a beam density of $10^{2} \mathrm{~m}^{-3}$ (see Lehtinen et al., 2001), or a charge removed (from $10 \mathrm{~km}$ altitude) of about $600 \mathrm{C}$. Optical emissions are expected to be delayed from the CG time, and the delay is defined by the bounce time for an energetic electron, which is weakly dependent on pitch angle (Walt, 1994). For $1 \mathrm{MeV}$ electrons, we expect a delay between the $+\mathrm{CG}$ and the conjugate emissions of about $60 \mathrm{~ms}$, corresponding to the propagation time at $L \simeq 1.78$ (location of SAAO).

\subsection{Instrumentation at $\mathrm{SAAO}$}

The Wide-angle Array for Sprite Photometry (WASP) is an array of six Hamamatsu HC104 photomultiplier tube assemblies (PMTs), as shown in Fig. 3. The PMTs are outfitted with lenses yielding a field-of-view of $\sim 3-$ by- $6^{\circ}$ for each element. The tubes are arranged with non-overlapping fieldsof-view, so that the resulting field-of-view is $\sim 6$-by- $16^{\circ}$ for the overall instrument. 
Each PMT is also fitted with a longpass optical filter with $665.0 \mathrm{~nm}$ cutoff, except one, which has a $3.0 \mathrm{~nm}$ bandwidth filter centered at $427.8 \mathrm{~nm}$, which is an $N_{2}^{+} 1 N$ emission line. The response curve of the PMTs is shown in Fig. 3, along with the response curves for the red filters, the emission band structure for the 1st positive band of molecular nitrogen $\left(N_{2} 1 P\right)$, and the atmospheric transmission curve for $N_{2} 1 P$.

The PMTs have built-in $8 \mathrm{MHz}$ bandwidth amplifiers. The signals from the PMTs are filtered at $10 \mathrm{kHz}$ and sampled at $25 \mathrm{kHz}$ per channel. Additionally, GPS timing signals are recorded with the data, as well as two channels of broadband $(300 \mathrm{~Hz}$ to $10 \mathrm{kHz})$ VLF data. The VLF receiver consists of orthogonal magnetic loop antennas, aligned with geographic North-South and East-West, fed into a preamplifier and signal-conditioning unit. This signal is then low-pass filtered with the WASP data at $10 \mathrm{kHz}$ and sampled at $25 \mathrm{kHz}$. Finally, video images overlapping the region viewed by the photometers is recorded using an intensified CCD camera for monitoring of local sky conditions.

\subsection{Coordination with sprite observations}

Sprite observations were made from the Observatoire du Pic du Midi (OMP) in the French Pyrenees, using two JAI CVS3200 cameras fitted with monochrome 1/2" Sony ExView CCDs. The system was set up to be remotely-controlled over the internet. Images that triggered the system were recorded and posted in real-time to the internet, along with lightning data provided by METEORAGE. The lightning data was additionally geomagnetically mapped to the conjugate region in real time, providing the locations of expected optical emissions. Local sprite and conjugate observations were coordinated whenever possible to give the best chance of seeing both events simultaneously.

Lightning is observed over Europe and mapped to the conjugate region using IGRF field models, updated annually, provided by NASA. The lightning data in Europe is mapped between $60 \mathrm{~km}$ altitudes, introducing some error into the location of the precipitation, which is added to the error inherent in drift variations of the Earth's magnetic field over the course of a year. These errors can add up to $20 \mathrm{~km}$, similar to the expected size of the precipitation region. However, the use of six non-overlapping fields-of-view ensures that the error is such that we can still be sure that most of the precipitation regions are within the field-of-view of the overall instrument.

\section{Description of data}

From 23 July to 29 August, $66 \mathrm{~h}$ of data were recorded with the WASP system, corresponding to thousands of $+\mathrm{CG}$ strokes in the European region of interest. When mapped to the conjugate region during recording times, over 1600 of these +CG's fell within the field-of-view of the instrument. These positive CG's ranged in intensity from $<10 \mathrm{kA}$ to $246 \mathrm{kA}$, with a mean of $37.1 \mathrm{kA}$.

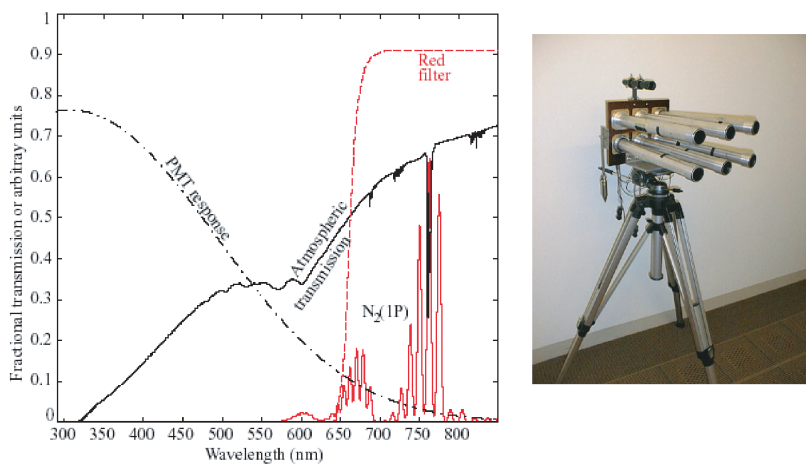

Fig. 3. Photometer response curve, atmospheric transmission, and filter response; and the WASP Instrument, consisting of six photomultiplier tubes aligned for a total Field-of-view of 6-by-16 ${ }^{\circ}$.

Sprite imaging data from OMP documented the occurrence of over 130 sprites during the same period, all associated with $+\mathrm{CG}$ lightning discharges. Of these, $30 \mathrm{oc}-$ curred during times and at locations for which WASP data was acquired, with at least one of the six WASP photometers viewing the altitudes in the conjugate region at which optical emissions would be expected.

No optical events associated with sprites or + CG's were found in the conjugate hemisphere. However, the 30 coordinated conjugate observations made constitute the first such optical observations in regions geomagnetically conjugate to sprites. We have associated our data with sprite observations because charge moments have been seen to be a good predictor of sprite occurrence (e.g., Hu et al., 2002); hence those CG's that produce sprites are the most likely to create relativistic beams. Two examples of observations associated with sprites are described below, along with an example of photometer response to a flashing airplane beacon.

\subsection{CASE 1: 28 August 2003, 23:41:54 UT}

Figure 4a shows an example of one-second of highresolution photometer data from 28 August 2003, at 23:41:54 UT, corresponding to an observed sprite in Europe. The time when we would expect to see optical emissions, if any were present, is shown in the figure for $1 \mathrm{MeV}$ electrons. Figure $4 \mathrm{~b}$ shows Nançay and Palmer VLF data corresponding to the causative $+\mathrm{CG}$. The Nançay data, only a few hundred $\mathrm{km}$ from the sprite location, exemplifies the intensity of the sferic, while the Palmer observation, at $13000 \mathrm{~km}$ distant, demonstrates the "slow-tail" effect of the sprite-causing $+\mathrm{CG}$, evidence of large ELF energy in the stroke (Reising et al., 1996). In Fig. 4c, the regions of interest are shown, with the sprite location marked on the top panel, and magnetically conjugated to the observation region, where the photometer fields-of-view are shown for a $55-\mathrm{km}$ altitude slice through the atmosphere. This mapping of the sprite location onto the photometer FOVs shows that any optical emissions 


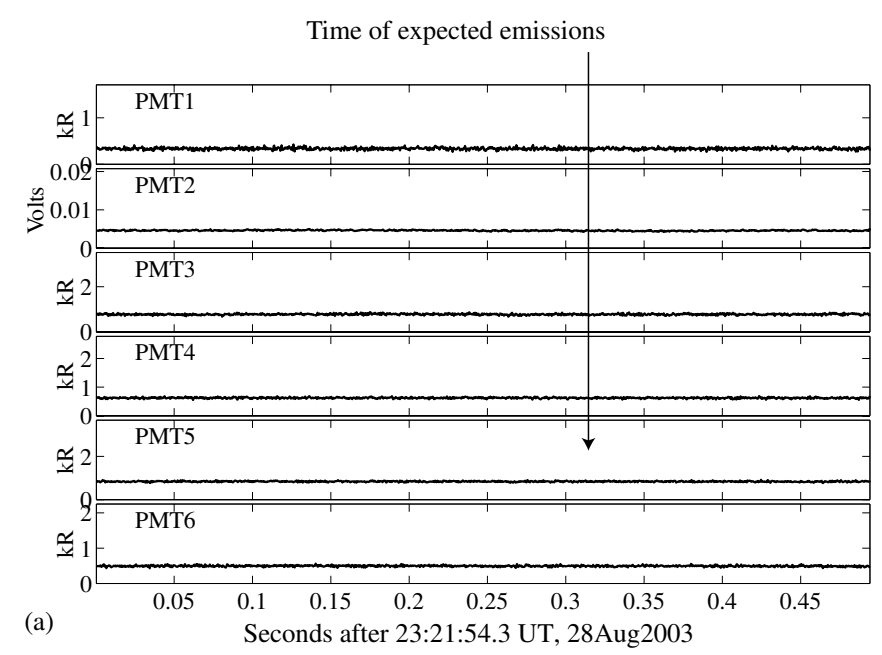

(b)

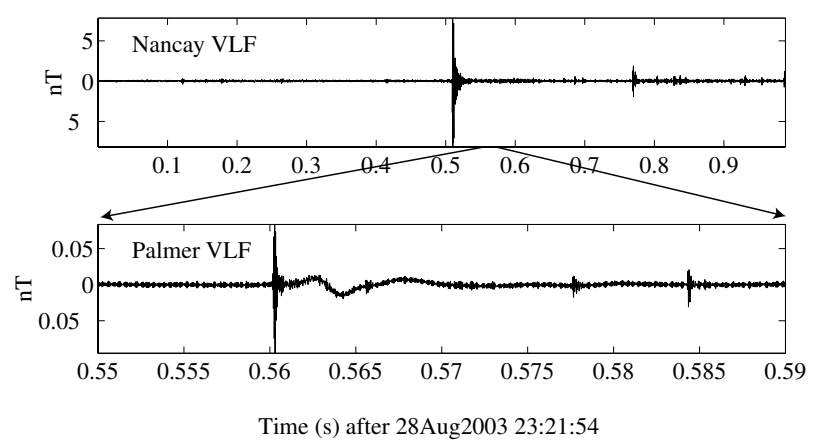

(e)

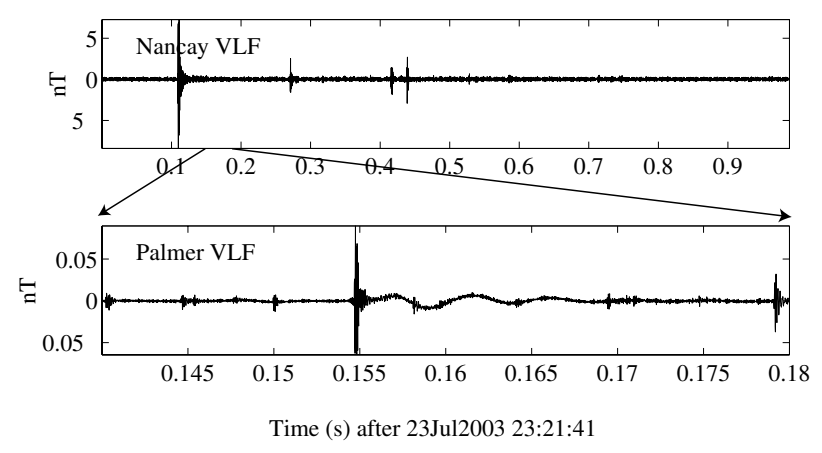

(d)

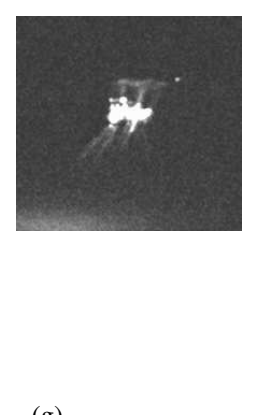

(g)

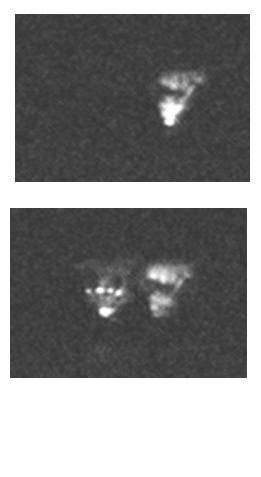

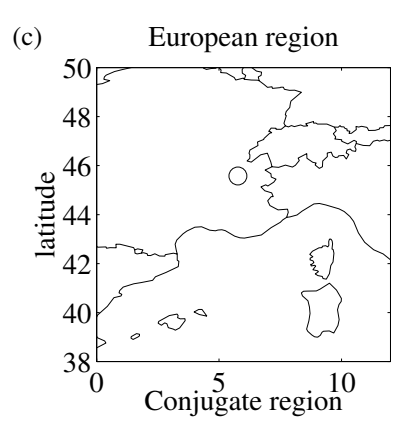
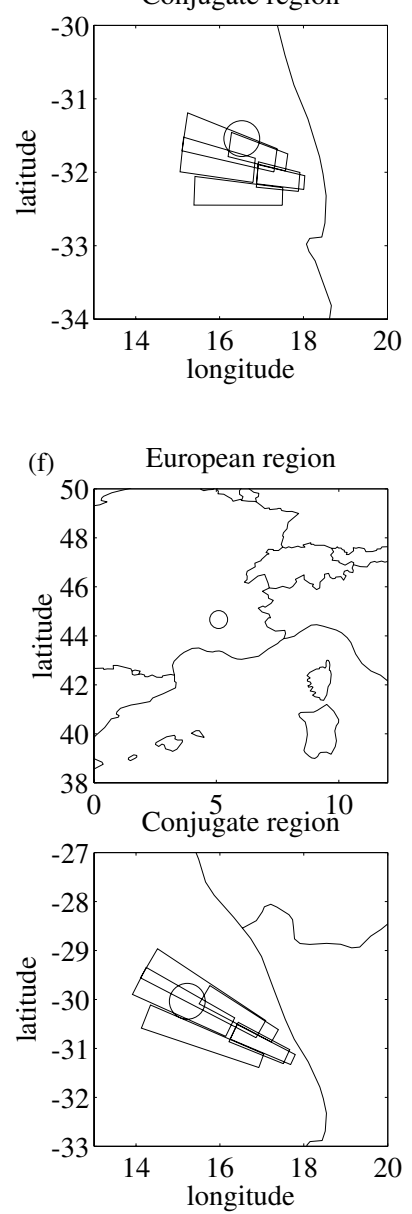

Fig. 4. Example data. (a) Photometer data from 28 August 2003, with time shown when $1 \mathrm{MeV}$ electrons would precipitate; (b) Corresponding Nançay and Palmer VLF data, where Palmer demonstrates the "slow-tail" of the sprite-causing +CG; (c) Corresponding regions of interest, showing location of sprite over Europe (circle), its location mapped to the conjugate region, and the photometers' field-of-view. (d) Corresponding sprite image. (e), (f) and (g) are equivalent to (b), (c) and (d) for an event of 23 July 2003. on 23 July 2003.

in the conjugate region due to precipitating energetic electrons would have fallen within the instrument field-of-view. Figure $4 \mathrm{~d}$ shows the sprite as observed from OMP.

\subsection{CASE 2: 23 July 2003, 23:21:41 UT}

Figure 4 also shows an example from 23 July 2003, at 23:21:41 UT. Photometer data is omitted to avoid redundancy. Figures $4 \mathrm{e}$, $\mathrm{f}$ and $\mathrm{g}$ correspond to the similar panels b, c, and d for the event of 28 August 2003. Again we note that the sprite location, when mapped to the conjugate region, falls within the instrument field-of-view, yet again, no emissions were detected.

\subsection{CASE 3: Airplane flash}

Figure 5 shows an example of photometer data from $6 \mathrm{Au}-$ gust 2003, at 18:13:37 UT, when an airplane was observed to pass through the instrument field-of-view. The top two panels show consecutive images from the wide field-of-view CCD camera, with a noticeable brightening visible in the second image as the airplane beacon is flashed. The $2-3 \mathrm{~ms}$ 
flash is very easily detected by the photometers despite the small area of brightening in comparison to the individual photometer field-of-view. This example is presented to show the working operation of the instrument in terms of its sensitivity in detecting small changes in optical intensity and its time resolution.

\section{Analysis}

The data examples in Figs. 4 and 5 show that any conjugate optical emissions must have been below the noise background of the nighttime sky, $\sim 1-3 \mathrm{kR}$ during the times shown. However, model predictions indicate that if the lightning discharges had large enough charge moments, peak emission intensities of up to $1 \mathrm{MR}$ may occur. Due to the highly nonlinear nature of the avalanche process, the most likely explanation for the lack of emissions is that none of the $+\mathrm{CG}$ 's considered created a large enough charge moment to create an appreciable relativistic beam.

This preliminary conclusion is supported by measurements of ELF sferic energy detected at Palmer Station, Antarctica, and by the charge moment calculations based on ELF observations at Nagycenk, Hungary, both of which are discussed below. By showing both ELF energies and charge moments for both Europe and the Midwestern U.S., we can verify the absolute charge moment values in the following way: ELF energies computed for strokes in Europe are compared to those in the U.S., and a difference is noted. Then, charge moments are compared to those in the U.S., and the difference is seen to be comparable.

\subsection{Charge moments}

Charge moments have been deduced from Schumann resonance (SR) transients detected in the vertical electric and horizontal magnetic field components in the frequency band of $5-30 \mathrm{~Hz}$ in the ELF $(3 \mathrm{~Hz}-3 \mathrm{kHz})$ range at Nagycenk, Hungary, during the EuroSprite2003 Campaign. The charge moments were derived from the current moment spectra by assuming exponentially decaying current in the time domain. Huang et al. (1999) showed that this assumption is particularly valid for sprite producing lightning flashes. Current moment spectra of the events processed here were estimated by comparing the measured spectra of the field components to the corresponding theoretical ones characteristic for the source distance (Huang et al., 1999). Theoretical spectra of the vertical electric and the horizontal magnetic field components were calculated using the Wait/Jones model in which the ground is considered perfectly conductive and the ionosphere is isotropic and characterized by the mean of three ionospheric conductivity profiles presented in Jones (1967). The parent lightning strokes of sprites, which can excite the Earth-ionosphere cavity resonator, can be modeled by a vertical electric dipole. Jones and Kemp (1970) showed that theoretical spectra of electromagnetic field components of a vertical electric dipole agree well with experimental results if

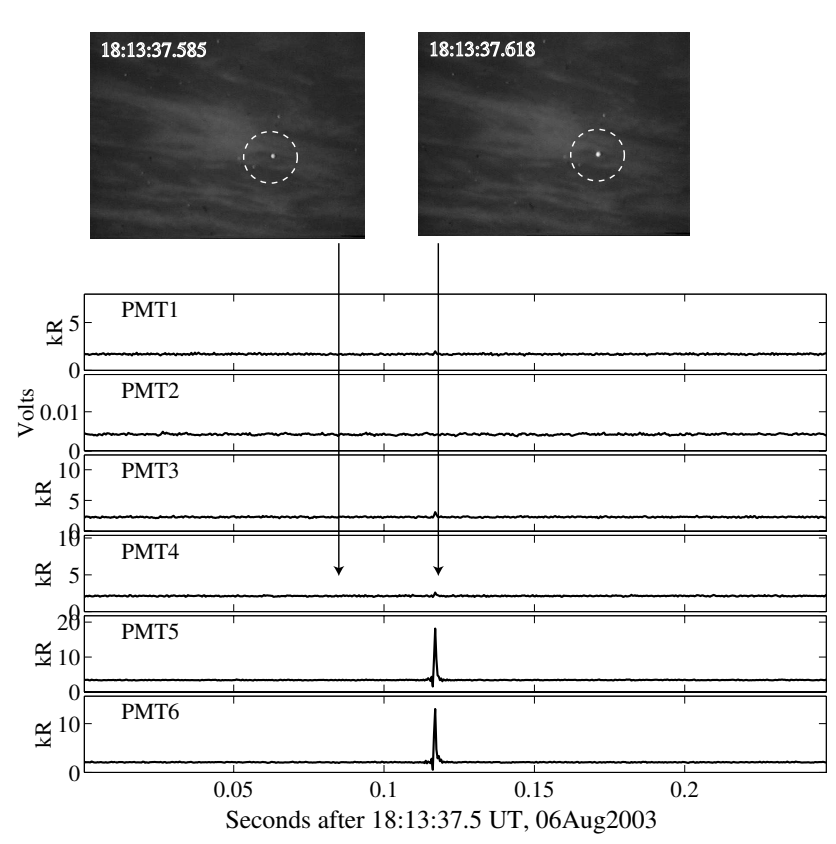

Fig. 5. Example data for 6 August 2003, demonstrating the effect of an airplane's beacon on the photometers' responses. The flash (circled in the images) appears in two photometers due to scattering from clouds.

they were calculated from formulae of Wait (1962) including the wave propagational parameters of Jones (1967).

Two methods were used to determine the charge moment from the evaluated current moment spectrum. Both methods are based on finding that exponential function Fourier transform of which is the best approximation to the current moment spectrum estimated. The charge moment is then calculated as the time integral of the determined exponential function (Burke and Jones, 1996; Huang et al., 1999).

The distribution of charge moments for 76 spriteproducing + CG's during the time of sprite observations in Europe is shown in Fig. 6. The largest of these charge moments is about $4100 \mathrm{C}-\mathrm{km}$, while the median is about $800 \mathrm{C}-\mathrm{km}$. The charge moment values derived here can be considered rough estimations due to the rather narrow bandwidth of the recording system and local electromagnetic disturbances.

A distribution of 23 typical charge moments in the Midwestern United States is also shown for comparison, taken from two storms on 2 July and 4 July 2000 . The charge moments from the U.S. are computed using a deconvolution method, as described in Cummer and Inan (2000). An impulse response for the Earth-Ionosphere waveguide propagation path between the lightning strike and the receiver is computed using the Long-Wave Propagation Capability (LWPC). The "output", the received signal, is then deconvolved with the LWPC impulse response to find the "input", which is the lightning current waveform.

We note that the charge moments are in general smaller for sprite-causing European strokes, when compared to 
(a) Europe, July/August 2003

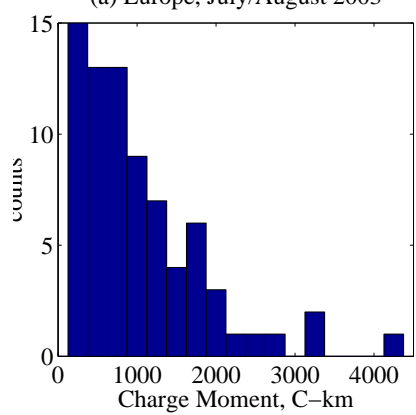

(b) North America, July 2/4 2000

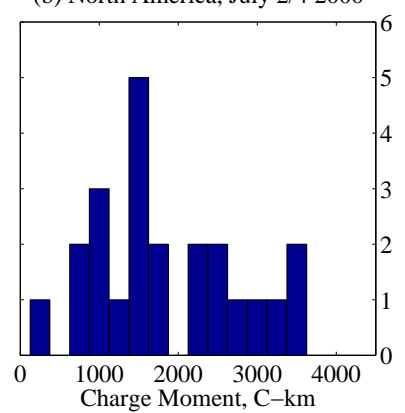

Fig. 6. Charge Moments calculations from (a) European $+\mathrm{CG}$ 's in 2003 and (b) Midwestern U.S. strikes from 2 and 4 July 2000. (a) Europe

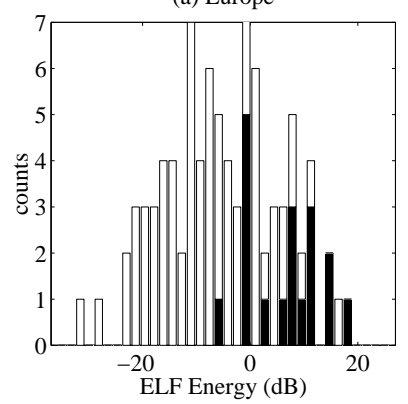

(b) North America

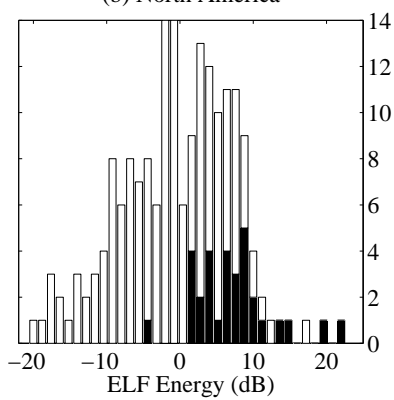

Fig. 7. ELF energy content of $+C G$ 's recorded at Palmer Station. (a) Europe 2003; (b) Midwestern United States, adapted from Reising et al. (1996). Shaded regions denote sprite-causing +CG's, while empty regions denote non-sprite-producing +CG's.

sprite-causing strokes in North America (median of about $1500 \mathrm{C}-\mathrm{km}$ ). Additionally, as mentioned above, the maximum charge moment found in Europe is only $4100 \mathrm{C}-\mathrm{km}$, smaller than the $6000 \mathrm{C}-\mathrm{km}$ predicted for detectable $(1 \mathrm{kR})$ emissions.

\subsection{Palmer ELF energy}

Reising et al. (1996) found that the ELF energy content of a $+\mathrm{CG}$ was a better measure of sprite occurrence than the peak currents typically recorded by a lightning detection network. While a well-established measure of the sprite-producing capacity of a lightning event is the charge moment (defined as the amount of charge removed multiplied by the vertical channel length), the ELF energy content gives a good measure of the continuing current in a $+\mathrm{CG}$, and it is this continuing current that is responsible for moving most of the charge from cloud to ground. Figure 7 shows a statistical comparison of typical ELF sferic energies is the Midwestern United States with those in Europe, as calculated at Palmer Station, Antarctica $\left(64^{\circ} 40^{\prime} \mathrm{S}, 60^{\circ} 0^{\prime} \mathrm{W}\right)$. The Stanford-operated VLF receiver system at Palmer Station consists of orthogonal magnetic field loop antennas, fed into an amplifier system with response from $350 \mathrm{~Hz}$ to $40 \mathrm{kHz}$ (see Fig. 4 for examples of Palmer data). ELF energies for sferics detected at
Palmer are calculated by low-pass filtering at $1.5 \mathrm{kHz}$ and integrating the square of the field amplitude from $2 \mathrm{~ms}$ before the sferic to $20 \mathrm{~ms}$ after, as in Reising et al. (1996). The difference in $0 \mathrm{~dB}$ reference values between Europe and North America is adjusted for propagation distance to Palmer by the following method. ELF attenuation rates increase with frequency, and are in the range from 0 to $5 \mathrm{~dB} / \mathrm{Mm}$ at frequencies from $1 \mathrm{~Hz}$ to $1 \mathrm{kHz}$ (Sukhorukov and Stubbe, 1997). The received signal at Palmer has a spectral peak at $200 \mathrm{~Hz}$; at this frequency the attenuation in the EarthIonosphere waveguide is about $2 \mathrm{~dB} / \mathrm{Mm}$ (Sukhorukov and Stubbe, 1997). The amplitudes of ELF energies received from Europe are shifted by this amount to compare them to ELF energies of North American lightning strikes. Even so, we see that the ELF content is statistically smaller for the European strikes than for the North American strikes, and is higher for sprite-causing strikes, as found previously (Reising et al., 1999). This supports the finding of statistically lower charge moments originating from Europe compared to those from North America.

\section{Summary and discussion}

We present the first optical measurements in the region geomagnetically conjugate to known sprite locations; these conjugate observations have been made coincident with about 30 sprites observed in Europe. Additionally, observations have been made coincident with over $1600+C G$ 's, for most of which simultaneous optical observations in the local region did not exist, so that the occurrence of sprites is not known.

Since the background noise of the night sky is $\sim 1 \mathrm{kR}$ in the wavelength band of interest, we conclude that any optical emissions in the conjugate hemisphere must have been below this noise level. It is highly likely that no discernible relativistic beam was created by any of the $1600+C G$ 's analyzed, and thus there were no optical emissions. The reason for this lack of a relativistic beam may be that the lightning strikes in Europe removed insufficient charge to create a dense enough runaway avalanche beam.

We have shown that the charge moments of the spriteproducing +CG's in Europe in 2003 do not exceed $4100 \mathrm{C}$ $\mathrm{km}$, while model predictions for the initiation of a relativistic beam have been conducted for a charge moment of $7500 \mathrm{C}$ $\mathrm{km}$. Figure 2 shows curves from Lehtinen et al. (2001) relating the beam density injected into the magnetosphere as a function of charge removed from clouds of different altitudes and shapes. Since optical emission intensities are linearly related to the precipitating beam density, $1 \mathrm{kR}$ of emissions (corresponding to the noise background) would require $N_{R} \geq 10^{2} \mathrm{~m}^{-3}$. Comparing this value to Fig. 2 , for a $10 \mathrm{~km}$ altitude point charge, a charge moment of $6000 \mathrm{C}-\mathrm{km}$ is required. This value still exceeds the largest charge moments seen in Europe during our experiment. Thus it is most probable that no +CGs analyzed removed enough charge to inject an observable electron beam. 
Acknowledgements. This work was supported by a Texas Instruments Stanford Graduate Fellowship, and ONR Grant N00014-03-1-0333. The authors would like thank the South African Astronomical Observatory for the use of its facility, and to METEORAGE for access to real-time lightning data. G. Sátori's and J. Bór's contribution was supported by Grants T034309 and TS 408048 from the Hungarian Science Foundation.

Topical Editor T. Pulkkinen thanks two referees for their help in evaluating this paper.

\section{References}

Burke, C. P. and Jones, D. L. I.: On the polarity and continuing currents in usually large lightning flashes deduced from ELF events, J. of Atmos. and Solar Terr. Phys., 58, 531-540, 1996.

Cummer, S. A. and Inan, U. S.: Modeling ELF radio atmospheric propagation and extracting lightning currents from ELF observations, Radio Sci., 35, 2, 385-394, 2000.

Dwyer, J. R., Rassoul, H. K., and Al-Dayeh, M., et al.: A ground level gamma-ray burst observed in association with rockettriggered lightning, Geophys. Res. Lett., 31, L05119, 2004.

Fishman, G. J., Bhat, P. N., and Malozzi, R., et al.: Discovery of intense gamma-ray flashes of atmospheric origin, Science, 264, 1313, 1994.

Hu, W., Cummer, S. A., Lyons, W. A., and Nelson, T. E.: Lightning charge moment changes for the initiation of sprites, Geophys. Res. Lett., 29, 8, 1279-1282, 2002.

Huang, E., Williams, E., Boldy, R., Heckman, S., Lyons, W., Taylor, M., Nelson, T., and Wong, C.: Criteria for sprites and elves based on Schumann resonance observations, J. of Geophys. Res., 104, D14, 16943-16964, 1999.
Inan, U. S., Reising, S. C., Fishman, G. J., and Horack, J. M.: On the association of terrestrial gamma-ray bursts with lightning and implications for sprites, Geophys. Res. Lett., 23, 9, 1017-1020, 1996.

Jones, D. L. I. and Kemp, D. T.: Experimental and theoretical observations of Schumann resonances, J. of Atmos. and Solar Terr. Phys., 32, 1095-1108, 1970.

Jones, D. L. I.: Schumann Resonances and e.l.f. propagation for inhomogeneous, isotropic ionosphere profiles, J. of Atmos. and Solar Terr. Phys., 29, 1037-1044, 1967.

Lehtinen, N. G., Inan, U. S., and Bell, T. F.: Effects of thunderstorm-driven runaway electrons in the conjugate hemisphere: purple sprites, ionization enhancements, and gamma rays, J. of Geophys. Res., 106, A12, 28 841-28 846, 2001.

Neubert, T., Allin, T. H., Stenbaek-Nielsen, H., and Blanc, E.: Sprites Over Europe, Geophys. Res. Lett., 28, 18, 3585-3588, 2001.

Reising, S. C., Inan, U. S., Bell, T. F., and Lyons, W. A.: Evidence for continuing current in sprite-producing cloud-to-ground lightning, Geophys. Res. Lett., 23, 24, 3639-3642, 1996.

Reising, S. C., Inan, U. S., and Bell, T. F.: ELF sferic energy as a proxy indicator for sprite occurrence, Geophys. Res. Lett., 26, 7, 987-990, 1999.

Sukhorukov, A. I. and Stubbe, P.: On ELF pulses from remote lightnings triggering sprites, Geophys. Res. Lett., 24, 13, 1639-1642, 1997.

Wait, J. R.: Electromagnetic Waves in Stratified Media, 2nd Ed. Pergamon Press, New York, 153, Sect. 5, 1962.

Walt, M.: Introduction to Geomagnetically Trapped Radiation, Cambridge University Press, 44, 1994. 